\title{
Knockdown of Fstl1 attenuates hepatic stellate cell activation through the TGF-ß31/Smad3 signaling pathway
}

\author{
HONGYE SHANG* ${ }^{*}$ XIANGJIN LIU* and HUI GUO \\ Digestive Department, First Teaching Hospital of Tianjin University of Traditional Chinese Medicine, Tianjin 300193, P.R. China
}

Received September 13, 2016; Accepted May 23, 2017

DOI: $10.3892 / \mathrm{mmr} .2017 .7445$

\begin{abstract}
Follistatin-like 1 (Fstl1) is a secreted glycoprotein that belongs to the follistatin and SPARC (secreted protein, acidic and rich in cysteine) families and was identified to serve a critical role in lung fibrosis. However, the role of Fstl1 in liver fibrosis remains undefined. Therefore, the aim of the present study was to investigate the role of Fstl1 in liver fibrosis. The results indicated that Fstl1 was highly expressed in human hepatic fibrosis tissues and activated hepatic stellate cells (HSCs). Furthermore, knockdown of Fstlleffectively suppressed HSC proliferation and the protein expression levels of $\alpha$-SMA and collagen I in transforming growth factor (TGF)- $\beta 1$-treated HSCs. Mechanistically, knockdown of Fstl1 remarkably decreased the phosphorylation level of Smad3 in TGF- $\beta 1$-induced HSCs. Taken together, the present study demonstrated that Fstl1serves an important role in liver fibrosis and target deletion of Fstl1 attenuated HSCs activation through suppressing TGF- $\beta 1 / \mathrm{Smad} 3$ signaling pathway. Therefore, Fstl1 may be a potential therapeutic target for the treatment of liver fibrosis.
\end{abstract}

\section{Introduction}

Liver fibrosis is the common consequence of chronic liver diseases and may progress to cirrhosis and hepatocellular carcinoma (HCC). It is characterized by accumulation of extracellular matrix (ECM) components, including collagen, fibronectin and laminin (1). The mechanisms underlying liver fibrosis are still largely unclear. Hepatic stellate cells (HSCs) are the predominant cell type in the development of liver fibrosis. The activation and transdifferentiation of HSCs are pivotal events in liver fibrosis $(2,3)$. Following liver

Correspondence to: Dr Hongye Shang, Digestive Department, First Teaching Hospital of Tianjin University of Traditional Chinese Medicine, 88 Changling Road, Tianjin 300193, P.R. China

E-mail: hongye_shang@126.com

${ }^{*}$ Contributed equally

Key words: follistatin-like 1, liver fibrosis, hepatic stellate cells, extracellular matrix injury, quiescent HSCs are exposed to different inflammatory cytokines, including transforming growth factor (TGF)- $\beta 1$, and then undergo a process of activation to a myofibroblastic phenotype, finally resulting in the excess production of ECM components (4). Thus, suppression of HSCs activation is the main approach for the treatment of liver fibrosis.

Follistatin-like 1 (Fstl1), also known as TSC-36, is a secreted glycoprotein that belongs to the follistatin and SPARC (secreted protein, acidic and rich in cysteine) families. Increasing evidences have reported that Fstl1servescritical roles in angiopoiesis, immunomodulation, embryonic development and tumorigenesis (5-8). For example, overexpression of FSTL1 significantly inhibited cell proliferation and invasion in ovarian and endometrial cancers (9). More recently, it was reported that Fstl1 is induced in response to lung injury; and blockage of Fstl1 with a neutralizing antibody attenuated bleomycin-induced lung fibrosis in vivo (10). However, the role of Fstl1 in liver fibrosis remains undefined. Therefore, the aim of the present study was to investigate the role of Fstl1 in liver fibrosis. The results demonstrated that knockdown of Fstl1 inhibited activation of HSCs through the TGF- $\beta 1 / \mathrm{Smad} 3$ signaling pathway.

\section{Materials and methods}

Specimen collection. Liver samples were collected by trans-parietal puncture from 11 healthy individuals and 11 patients with liver fibrosis. Written informed consent was obtained from all patients, and the study was approved by the Medical Ethics Committee of First Teaching Hospital of Tianjin University of Traditional Chinese Medicine (Tianjin, China).

Cell culture. Primary HSCs were isolated as described previously (11). Cells were cultured in Dulbecco's modified Eagle's medium (DMEM; Invitrogen; Thermo Fisher Scientific, Inc., Waltham, MA, USA) supplemented with $10 \%(\mathrm{v} / \mathrm{v})$ fetal bovine serum (FBS; Gibco; Thermo Fisher Scientific, Inc.), L-glutamine (4 mmol/l; Sigma-Aldrich; Merck KGaA, Darmstadt, Germany), penicillin (100 IU/ml; Sigma-Aldrich; Merck KGaA), and streptomycin (100 $\mu \mathrm{g} / \mathrm{ml}$; Sigma-Aldrich; Merck $\mathrm{KGaA}$ ) at $37^{\circ} \mathrm{C}$ in a humidified $5 \% \mathrm{CO}_{2}$ atmosphere.

RNA interference and cell transfection. Small-interfering RNA targeting Fstl1 (si-Fstl1) and non-targeting control siRNA 
(scramble) were synthesized by Guangzhou Ribo Bio Co., Ltd. (Guangzhou, China). HSCs at a density of $5 \times 10^{4}$ cells/well were seeded in each cell of a 24-well micro-plate, grown for $24 \mathrm{~h}$ to reach $30-50 \%$ confluence, and then transfected with si-Fstl1 or scramble using Lipofectamine 2000 (Invitrogen; Thermo Fisher Scientific, Inc.) according to the manufacturer's instructions.

Reverse transcription-quantitative polymerase chain reaction $(R T-q P C R)$. Total RNA of quiescent HSCs and activated HSCs, as well as normal and hepatic fibrosis tissues, was extracted and purified using an RNeasy Mini kit according to the instructions of the manufacturer (Qiagen, Inc., Valencia, CA, USA). Up to $5 \mu \mathrm{g}$ of the total RNA was reverse-transcribed into cDNA using M-MLV reverse transcriptase (Sigma-Aldrich; Merck KGaA). RT-qPCR was performed with the Applied Biosystems 7900HT Fast Real-Time PCR System (Applied Biosystems; Thermo Fisher Scientific, Inc.) using a SYBR Green Real-Time PCR Master Mix kit (Takara Biotechnology Co., Ltd., Dalian, China). The following primers were used: Fstl1, 5'-CGAGGT GGAGTTGACGAGAAAC-3' (sense), 5'-AGGACTGGAT CATCATGACGTTCT-3' (antisense); and $\beta$-actin 5'-CCGTGA AAAGATGACCCAGATC-3' (sense), 5'-CACAGCCTGGAT GGCTACGT-3' (antisense). The relative levels of transcript were determined by using the $2^{-\Delta \Delta C q}$ method (12) and normalized by $\beta$-actin.

Western blotting. Total protein was extracted from hepatic fibrosis tissues or HSCs using RIPA Cell Lysis buffer (Takara Biotechnology Co., Ltd.). Lysates were sonicated for $5 \mathrm{sec}$ on ice and centrifuged at $6,000 \mathrm{xg}$ for $5 \mathrm{~min}$ at $4^{\circ} \mathrm{C}$. Supernatants were collected and the protein concentration was detected using a Bio-Rad Protein Assay kit II (cat. no. 500-0002; Bio-Rad Laboratories, Inc., Hercules, CA, USA). The cell lysates (30 $\mu \mathrm{g} / \mathrm{lane}$ ) were subjected to SDS-PAGE and subsequently transferred to a polyvinylidene difluoride membrane. Then, nonspecific binding was blocked by incubating with $5 \%$ non-fat milk in TBS containing $0.1 \%$ Tween- 20 at room temperature for $1 \mathrm{~h}$. The blots were incubated with primary antibodies: anti-Fstl1 (1:1,000; cat. no. sc-80408), anti-collagen I (1:2,000; cat. no. sc-59772), anti- $\alpha$-SMA (1:2,000; cat. no. sc-130616), anti-Smad3 (1:2,000; cat. no. sc-101154), anti-p-Smad3 $(1: 2,000$; cat. no. sc-11769) or anti-GAPDH (1:5,000; cat. no. sc-400163; all from Santa Cruz Biotechnology, Inc., Dallas, TX, USA) at $4^{\circ} \mathrm{C}$ overnight. Subsequently, the membranes were incubated with horseradish peroxidase-conjugated secondary antibodies (1:2,000; cat. no. sc-2789; Santa Cruz Biotechnology, Inc.) for $1 \mathrm{~h}$ at room temperature. Following washing, the blots were visualized using an enhanced chemiluminescence detection system (GE Healthcare Life Sciences, Chalfont, UK). Protein expression was analyzed using BandScan software version 5.0 (Glyko; BioMarin Pharmaceutical, Inc., San Rafael, CA, USA). All experiments were repeated $\geq 3$ times.

Cell proliferation assay. Cell proliferation was determined using the Cell Counting kit-8 assay. Briefly, infected HSCs were plated at a density of $1 \times 10^{4}$ cells/well in a 96 -well culture plate and treated with or without TGF- $\beta 1$ for $24 \mathrm{~h}$. Then, $10 \mu \mathrm{lCCK}-8$ reagent was added (Dojindo Molecular Technologies, Inc., Kumamoto, Japan) to each well. Following $1 \mathrm{~h}$ of incubation at
A

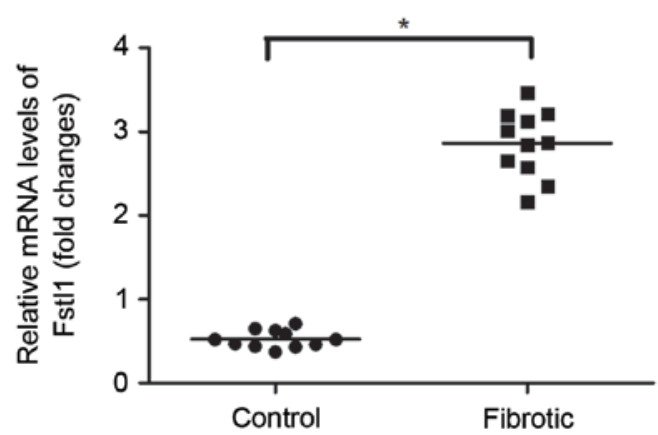

B

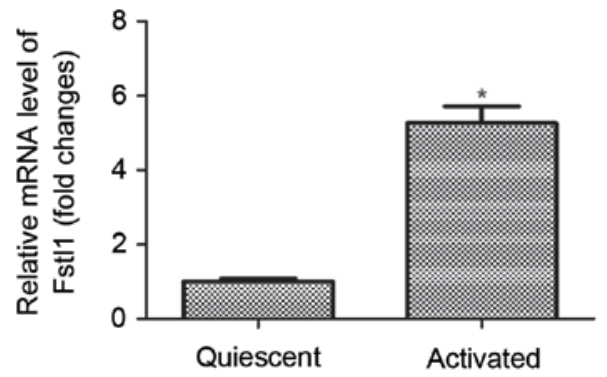

C
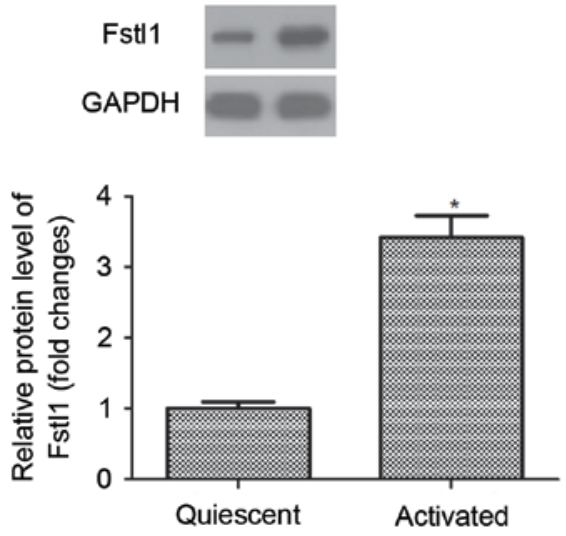

Figure 1. Fstl1 is highly expressed in hepatic fibrosis tissues and activated HSCs. (A) The mRNA levels of Fstl1 were significantly higher in human fibrotic liver tissues compared with human normal liver tissues. ${ }^{*} \mathrm{P}<0.05$ as indicated. (B) The mRNA levels of Fstl1 were detected using reverse transcription-quantitative polymerase chain reaction in quiescent and activated HSCs. (C) The protein levels of Fstl1 were detected using western blotting in quiescent and activated HSCs. ${ }^{*} \mathrm{P}<0.05$ vs. quiescent cells. Data represents the mean \pm standard deviation of three independent experiments. Fst1, follistatin-like 1; HSCs, hepatic stellate cells.

$37^{\circ} \mathrm{C}$, the absorbance was measured with a microplate reader (Bio-Rad Laboratories, Inc.) at a wavelength of $490 \mathrm{~nm}$.

Statistical analysis. All statistical analyses were performed using the SPSS software (version, 13.0; SPSS, Inc., Chicago, IL, USA). Results were presented as mean \pm standard deviation for three experiments. Statistical comparisons were performed using one-way analysis of variance followed by the Student's t-test. $\mathrm{P}<0.05$ was considered to indicate a statistically significant difference.

\section{Results}

Fstll is highly expressed in hepatic fibrosis tissues and activated HSCs. The authors initially measured the expression of 
A

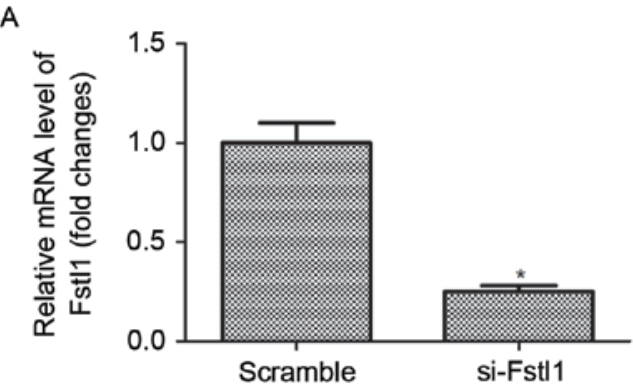

B

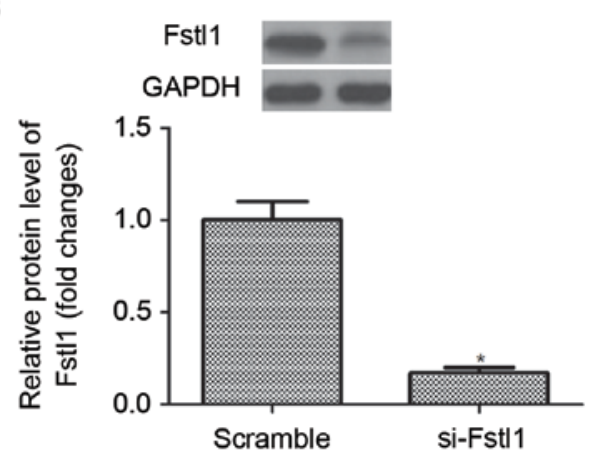

C

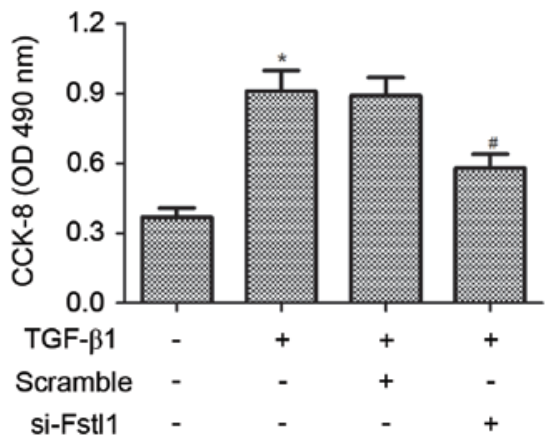

Figure 2. Knockdown of Fstl1 inhibits the proliferation of activated HSCs. HSCs were transfected with si-Fstll or scramble, respectively, for $24 \mathrm{~h}$. (A) The mRNA level of Fstl1 was detected using reverse transcription-quantitative polymerase chain reaction in quiescent and activated HSCs. (B) The protein level of Fstll was examined using western blotting assay. Then cells were treated with TGF- $\beta 1(10 \mathrm{ng} / \mathrm{ml})$ for $24 \mathrm{~h}$. (C) Cell proliferation was evaluated by the CCK- 8 assay. Data represents the mean of three independent experiments \pm standard deviation. ${ }^{~} \mathrm{P}<0.05$ vs. control group; ${ }^{\#} \mathrm{P}<0.05$ vs. TGF- $\beta 1+$ scramble group. Fst1, follistatin-like 1 ; HSCs, hepatic stellate cells; TGF- $\beta 1$, transforming growth factor- $\beta 1$.

Fstl1 in human hepatic fibrosis tissues. As indicated in Fig. 1A, the mRNA expression levels of Fstl1 were significantly upregulated in human hepatic fibrosis tissues, as compared with the normal liver tissues. Moreover, the authors observed that the expression levels of Fstll at both mRNA and protein were higher in activated HSCs than that of in the quiescent cells (Fig. 1B and C).

Knockdown of Fstll inhibits the proliferation of activated HSC. In order to investigate the effect of Fstll on HSC proliferation, HSCs were transfected with si-Fstll or scramble, respectively. The efficiency of transfection was confirmed by RT-qPCR and western blotting. The results of RT-qPCR analysis indicated that the mRNA expression of Fstl1 was obviously decreased in HSCs transfected with si-Fstl1 (Fig. 2A). Similarly, knockdown of Fstl1 greatly downregulated the protein expression level
A

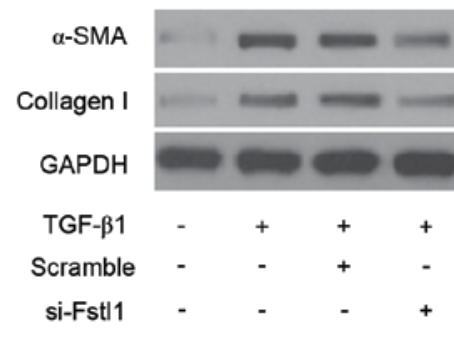

B

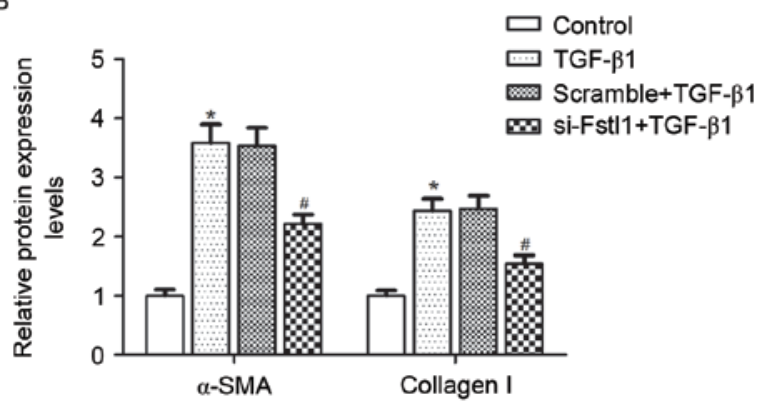

Figure 3. Knockdown of Fstll inhibits the expression levels of $\alpha$-SMA and collagen I in HSCs. HSCs were transfected with si-Fstl1 or scramble, respectively, for $24 \mathrm{~h}$. Then cells were treated with TGF- $\beta 1(10 \mathrm{ng} / \mathrm{ml})$ for $24 \mathrm{~h}$. (A) The protein expression levels of $\alpha$-SMA and type I collagen were examined using western blotting assay. (B) Quantification analysis was performed using Gel-Pro Analyzer version 4.0 software. Data represents the mean of three independent experiments \pm standard deviation. ${ }^{*} \mathrm{P}<0.05$ vs. control group; ${ }^{\#} \mathrm{P}<0.05$ vs. TGF- $\beta 1+$ scramble group. Fst1, follistatin-like $1 ; \alpha$-SMA, $\alpha$-smooth muscle actin; HSCs, hepatic stellate cells; TGF- $\beta 1$, transforming growth factor- $\beta 1$.

A

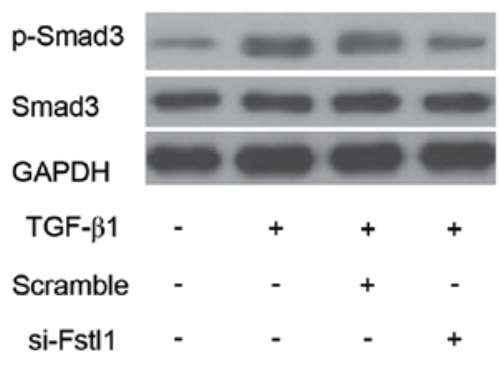

B

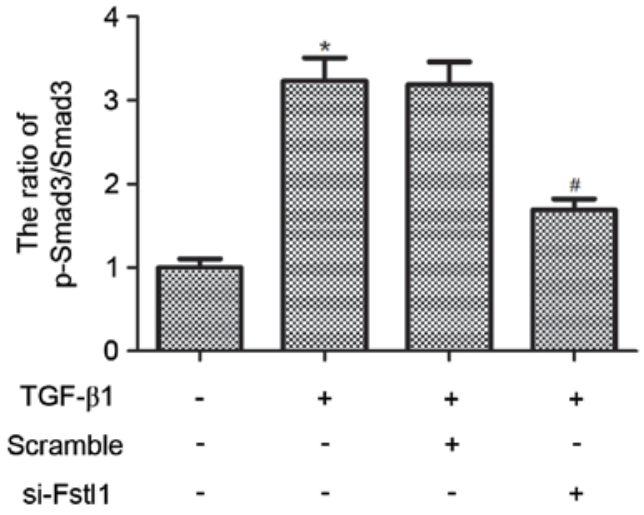

Figure 4. Fst11 is involved in the regulation of TGF- $\beta 1$-mediated Smad2/3 signaling pathway in HSCs. HSCs were transfected with si-Fst11 or scramble, respectively, for $24 \mathrm{~h}$. Then cells were treated with TGF- $\beta 1(10 \mathrm{ng} / \mathrm{ml})$ for 30 min. (A) The protein expression levels of $\mathrm{p}-\mathrm{Smad} 3$ and Smad3 were detected by western blot analysis. (B) Quantification analysis was performed using Gel-Pro Analyzer version 4.0 software. Data represents the mean of three independent experiments \pm standard deviation. ${ }^{*} \mathrm{P}<0.05$ vs. control group; ${ }^{\sharp} \mathrm{P}<0.05$ vs. TGF- $\beta 1+$ scramble group. Fst1, follistatin-like 1 ; TGF- $\beta 1$, transforming growth factor- $\beta 1$; HSCs, hepatic stellate cells. 
of Fstl1 in HSCs (Fig. 2B). Then, the authors performed the CCK-8 assay to detect the effect of Fstll on HSC proliferation. As indicated in Fig. 2 C, TGF- $\beta 1$ treatment markedly promoted the proliferation of HSCs, compared with the control group. However, knockdown of Fstl1 significantly inhibited HSC proliferation in TGF- $\beta 1$-induced HSCs.

Knockdown of Fstl1 inhibits the expression levels of $\alpha$-SMA and collagen $I$ in HSCs. The authors next investigated whether si-Fstl1 attenuated ECM expression in HSCs. The results of western blotting analysis demonstrated that TGF- $\beta 1$ obviously induced the protein expression levels of $\alpha$-SMA and type I collagen, as compared with the control group. Meanwhile, knockdown of Fstl1 significantly blunted TGF- $\beta 1$-induced $\alpha$-SMA and type I collagen expression in HSCs (Fig. 3).

Fstl1 is involved in the regulation of TGF- $\beta 1$-mediated Smad3 signaling pathway in HSCs. To determine the molecular mechanism of Fstl1 regulates HSCs activation described above, the effect of Fstll on the activation of Smad3 signaling pathway in HSCs was examined. Western blotting indicated that TGF- $\beta 1$ led to a marked increase in Smad3 phosphorylation; however, knockdown of Fstl1 remarkably decreased the phosphorylation level of Smad2/3 in TGF- $\beta 1$-induced HSCs (Fig. 4).

\section{Discussion}

In the current study, the results indicated that Fstll was highly expressed in human hepatic fibrosis tissues and HSCs. Furthermore, knockdown of Fstlleffectively suppressed HSC proliferation and the protein expression levels of $\alpha$-SMA and collagen I in TGF- $\beta 1$-treated HSCs. Mechanistically, knockdown of Fstllremarkably decreased the phosphorylation level of Smad3 in TGF- $\beta 1$-induced HSCs.

Prior studies have documented that Fstll contributes to the fibrogenesis. Maruyama et al (13) confirmed that the expression of Fstl1 was upregulated in ischemic portions of the myocardium, and the reparative fibrotic response conferred by Fstll is the consequence of its early activation of cardiac fibroblasts that leads to myofibroblast accumulation in the infarct area. Murphy et al (14) reported that the protein expression level of Fstl1 was greatly increased in the lungs of bleomycin-treated mice and in the lungs of idiopathic pulmonary fibrosis patients. In agreement with the results above, herein, the authors observed that Fstll is overexpressed in human liver fibrotic tissues comparing with the normal liver tissues, and the expression of Fstll was higher in activated HSCs than that of quiescent HSCs. These findings suggested that Fstl1 servesa pro-fibrotic role in liver fibrosis.

HSC activation is the primary feature during the progression of liver fibrosis; these activated HSCs increase proliferation and migration, and acquire contractility and pro-inflammatory properties (15). The induction of HSCs proliferation is stimulated by a variety of cytokines (16-18). TGF- $\beta 1$ is the most potent stimulus for HSC-mediated fibrogenesis (19). Herein, the authors observed that TGF- $\beta 1$ treatment markedly promoted the proliferation of HSCs. However, knockdown of Fstll significantly inhibited HSC proliferation.
Liver fibrosis is characterized by an exacerbated accumulation of deposition of ECM proteins (20). There is extensive evidence demonstrating that TGF- $\beta 1$ upregulated expression of $\alpha$-SMA and collagen I in HSCs (21-23). Recently, it was reported that the expression of $\alpha$-SMA, type I collagen and fibronectin in fibroblasts isolated from bleomycin-treated $\mathrm{Fstll}^{+/-}$lungs was markedly decreased (10). Similarly, in the present study, knockdown of Fstll effectively suppressed the protein expression levels of $\alpha$-SMA and collagen I in TGF- $\beta 1$-treated HSCs. These data suggested that knockdown of Fstl1 inhibited HSC activation by downregulating the protein expression levels of $\alpha$-SMA and collagen I in TGF- $\beta 1$-treated HSCs.

The TGF- $\beta 1 /$ Smad signaling pathway serves an important role in the development of liver fibrosis (24-26). During fibrogenesis, TGF- $\beta 1$ exerts its biological functions via a heteromeric receptor complex of type II and type I receptor serine-threonine kinases. Subsequently, Smad2/3 is phosphorylated and binds with Smad4 to form multimeric complexes, then activated R-Smads translocate to the nucleus and induce the expression of target genes, including ECM proteins (27). It has been reported that knockdown of Smad3 significantly reduced TGF- $\beta 1$-induced collagen I production in HSCs (28). In the present study, the authors indicated that TGF- $\beta 1$ led to a marked increase in Smad3 phosphorylation; however, knockdown of Fstl1 remarkably decreased the phosphorylation level of Smad3 in TGF- $\beta 1$-induced HSCs. These data suggested that Fstl1 silencing inhibits HSCs activation through suppressing the TGF- $\beta 1 /$ Smad3 signaling pathway.

In conclusion, it has been demonstrated that Fstl1servesan important role in liver fibrosis and target deletion of Fstl1 attenuated HSCs activation through suppressing the TGF- $\beta 1 /$ Smad 3 signaling pathway. Therefore, Fstl1 may be a potential therapeutic target for the treatment of liver fibrosis.

\section{References}

1. Friedman SL: Mechanisms of hepatic fibrogenesis. Gastroenterology 134: 1655-1669, 2008.

2. Bataller R and Brenner DA: Hepatic stellate cells as a target for the treatment of liver fibrosis. Semin Liver Dis 21: 437-451, 2001.

3. Reeves HL and Friedman SL: Activation of hepatic stellate cells-a key issue in liver fibrosis. Front Biosci 7: d808-d826, 2002.

4. Inagaki Y and Okazaki I: Emerging insights into transforming growth factor beta Smad signal in hepatic fibrogenesis. Gut 56: 284-292, 2007.

5. Ouchi N, Oshima Y, Ohashi K, Higuchi A, Ikegami C, Izumiya $\mathrm{Y}$ and Walsh K: Follistatin-like 1, a secreted muscle protein, promotes endothelial cell function and revascularization in ischemic tissue through a nitric-oxide synthase-dependent mechanism. J Biol Chem 283: 32802-32811, 2008.

6. Kudo-Saito C: FSTL1 promotes bone metastasis by causing immune dysfunction. Oncoimmunology 2: e26528, 2013.

7. Adams D, Larman B and Oxburgh L: Developmental expression of mouse Follistatin-like 1 (Fst11): Dynamic regulation during organogenesis of the kidney and lung. Gene Expr Patterns 7: 491-500, 2007.

8. Kudo-Saito C, Fuwa T, Murakami K and Kawakami Y: Targeting FSTL1 prevents tumor bone metastasis and consequent immune dysfunction. Cancer Res 73: 6185-6193, 2013.

9. Chan QK, Ngan HY, Ip PP, Liu VW, Xue WC and Cheung AN: Tumor suppressor effect of follistatin-like 1 in ovarian and endometrial carcinogenesis: A differential expression and functional analysis. Carcinogenesis 30: 114-121, 2009. 
10. Dong Y, Geng Y, Li L, Li X, Yan X, Fang Y, Li X, Dong S, Liu X, Li X, et al: Blocking follistatin-like 1 attenuates bleomycin-induced pulmonary fibrosis in mice. J Exp Med 212: 235-252, 2015

11. Chang W, Yang M, Song L, Shen K, Wang H, Gao X, Li M, Niu W and Qin X: Isolation and culture of hepatic stellate cells from mouse liver. Acta Biochim Biophys Sin (Shanghai) 46: 291-298, 2014.

12. Livak KJ and Schmittgen TD: Analysis of relative gene expression data using real-time quantitative PCR and the 2(-Delta Delta C(T)) method. Methods 25: 402-408, 2001.

13. Maruyama S, Nakamura K, Papanicolaou KN, Sano S, Shimizu I, Asaumi Y, van den Hoff MJ, Ouchi N, Recchia FA and Walsh K: Follistatin-like 1 promotes cardiac fibroblast activation and protects the heart from rupture. EMBO Mol Med 8: 949-966, 2016

14. Murphy N, Gaynor KU, Rowan SC, Walsh SM, Fabre A Boylan J, Keane MP and McLoughlin P: Altered expression of bone morphogenetic protein accessory proteins in murine and human pulmonary fibrosis. Am J Pathol 186: 600-615, 2016.

15. Svegliati-Baroni G, Ridolfi F, Hannivoort R, Saccomanno S Homan M, De Minicis S, Jansen PL, Candelaresi C, Benedetti A and Moshage $\mathrm{H}$ : Bile acids induce hepatic stellate cell proliferation via activation of the epidermal growth factor receptor. Gastroenterology 128: 1042-1055, 2005

16. Khan F,Peltekian KM and Peterson TC: Effect of interferon-alpha, ribavirin, pentoxifylline, and interleukin-18 antibody on hepatitis $\mathrm{C}$ sera-stimulated hepatic stellate cell proliferation. J Interferon Cytokine Res 28: 643-651, 2008

17. Bendia E, Benedetti A, Baroni GS, Candelaresi C, Macarri G, Trozzi L and Di Sario A: Effect of cyanidin 3-O-beta-glucopyranoside on hepatic stellate cell proliferation and collagen synthesis induced by oxidative stress. Dig Liver Dis 37: 342-348, 2005.

18. Li J, Si HF, Lü X, Guo A and Jiang H: Suppressive effects of leflunomide on leptin-induced TIMP-1 production involves on hepatic stellate cell proliferation and apoptosis. Eur J Pharmacol 580: 63-69, 2008.
19. He Y,Huang C,Sun X,Long XR,Lv XW and Li J: MicroRNA-146a modulates TGF-beta1-induced hepatic stellate cell proliferation by targeting SMAD4. Cell Signal 24: 1923-1930, 2012.

20. Bataller R and Brenner DA: Liver fibrosis. J Clin Invest 115 209-218, 2005.

21. Wiercinska E, Wickert L, Denecke B, Said HM, Hamzavi J, Gressner AM, Thorikay M, ten Dijke P, Mertens PR, Breitkopf K and Dooley S: Id1 is a critical mediator in TGF-beta-induced transdifferentiation of rat hepatic stellate cells. Hepatology 43: 1032-1041, 2006.

22. He P, Yu ZJ, Sun CY, Jiao SJ and Jiang HQ: Knockdown of eIF3a attenuates the pro-fibrogenic response of hepatic stellate cells induced by TGF- $\beta 1$. Cell Mol Biol (Noisy-le-grand) 62: 107-111, 2016.

23. Ma HH, Yao JL, Li G, Yao CL, Chen XJ and Yang SJ: Effects of c-myb antisense RNA on TGF-beta1 and beta1-I collagen expression in cultured hepatic stellate cells. World J Gastroenterol 10: 3662-3665, 2004

24. Luo JY, Zhang LT, Ming-Xia LU, Yan XM, Xiao P and Medicine DO: Smurf 2 impact on TGF- $\beta /$ Smad pathway in liver fibrosis. Med Recapitulate 17: 125-131, 2015.

25. Zhou Y, Tong X, Ren S, Wang X, Chen J, Mu Y, Sun M, Chen G, Zhang H and Liu P: Synergistic anti-liver fibrosis actions of total astragalus saponins and glycyrrhizic acid via TGF- $\beta 1 /$ Smads signaling pathway modulation. J Ethnopharmacol 190: 83-90, 2016.

26. Yang JW and Wook K: The role of Pin 1 in liver fibrosis: Regulation of Tgf- $\beta 1$ expression and Smad2/3 phosphorylation. Toxicol Lett 229 (Suppl): S240, 2014.

27. Breitkopf $K$, Weng $H$ and Dooley S: TGF- $\beta /$ Smad-signaling in liver cells: Target genes and inhibitors of two parallel pathways. Signal Transduction 6: 329-337, 2006.

28. Zhang L, Liu C, Meng XM, Huang C, Xu F and Li J: Smad2 protects against TGF- $\beta 1 / \mathrm{Smad} 3$-mediated collagen synthesis in human hepatic stellate cells during hepatic fibrosis. Mol Cell Biochem 400: 17-28, 2015. 